[Review]

\title{
Epidemiological consideration on the difference in frequency of apoplexy and coronary heart disease in Japan compared with European countries.
}

\section{Hiroshi OKADA*}

It is well known that in Japan the mortality rate from cerebrovascular disease has been very high, though that from cardiovascular disease has been fairly low. Today this tendency has not changed.

However, there is seen a little decrease in cerebrovascular death and a little inc. rease in cardiovascular death.

I should like to present the current features of these two diseases in Japan, and comparing them with those in European countries, to consider the cause from an epidemiological point of view. ${ }^{1,2), 3)}$

Since 1947, the first cause of death in Japan has been cerebrovascular disease and the third, cardiovascular disease, contrary to European countries.

Figure 1 shows the age-adjusted death rate from cerebrovascular disease in $\mathrm{Ja}$. pan compared with 10 other countries. ${ }^{4), 5,6)}$ The rate in Japan is the highest for both males and females in 1971.

By contrast, Figure 2 shows the ageadjusted death rate from cardiovascular disease in 12 countries. ${ }^{4), 7)}$ Japan is the lowest both for males and females.

Figured 3 shows the mortality trend of cerebro- and cardiovascular diseases in Japan since te beginning of 20 th cent. ury. ${ }^{4)}$ Recently cerebrovascular disease has shown a little decrease, but reversely cardiovascular disease a little increase.

The characteristic feature of cerebrovascular disease in Japan lies in the higher incidence of cerebralhemorrhage than in European countries. Figure 4 showe the ratio of cerebralhemorrhage to cerebralthrombosis by population surveys in Japan, U.S.A. and Australia. ${ }^{8-131}$ In Japan this ratio is much higher than in other countries. This feature is also seen in autopsy data of Japan and European countries.

As the causes of this high mortality from cerebrovascular disease in Japan. The way of living, structure of houses, diet, etc., could be considered.

I should like to mention especially the structure of housing and high salt intake to a monotonous rice diet.

Figure 5 indicates a regional difference of age-adjusted death rate from cerebrovascular disease. ${ }^{14)}$ It shows a higher mortality in the northern part, except for Hokkaido, the northern most is land.

In Japan even today many homes are. tropical style in structure, with the room temperature almost the same as that outdoor, so that the residents are exposed to the cold in winter. However, in Hokkaido 
OKADA : Epidemiological consideration on the difference in frequency of apoplexy and coronary heart disease in Japan compared with European countries.

the houses are northern in type and have been equipped with heating, ever since the island was settled at the end of the 19 th century.

Figure 6 shows the relationship of salt intake with cerebral stroke. ${ }^{15)}$ You will see a high correlation between the two. The high consumption of salt is an inevitable results of a monotonous diet consisting of a high percentage of rice. Such relation exists also between hyper tension and salt intake.

Figure 7 shows the regional difference of age-adjusted death rate from cardiovascular disease. ${ }^{14)}$ No significant regional difference is seen when compared with that of cerebrovascular disease.

Figure 8 shows that cardiovascular death has been increasing, especially in metropolitan cities than in other cities and rural ditricts. ${ }^{4)}$

Figure 9 shows that trend of the diet in recent years in Japan. ${ }^{16)}$ The total calories intake has increased most in rural districts due to a higher intake of carebohydrates. In contrast, the intake of fats is greatest in metropolitan cities due to the diet becoming more western in style.

Figure 10 shows the average serum cholesterol level of the Japanese for all ages. ${ }^{17}$ You will see the level is highest in metropolitan areas. though it is still fairly low when compared with that of European countries.

In concluding, I would like to stress that what will be the most important problem in Japan, is to decrease the incidence of cerebrovascular disease andalso to prevent an increase in cradiovascular disease.
Figure 1. Age-Adjusted Death Rate from Cardiovascular Disease in 12 Countries, 1970 $1970 \cdot 1971$ (per 100,000 population

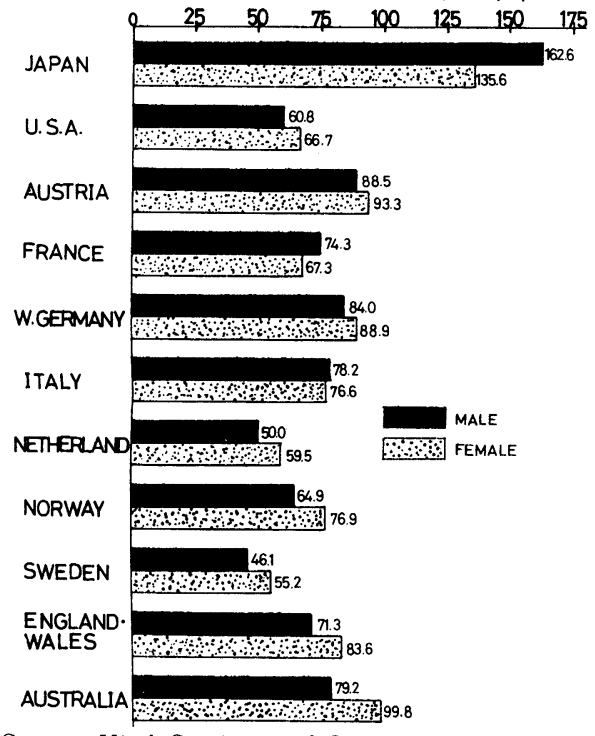

Source :Vital Statistics of Japan.

World Health Statistics Annual 1971 Vol.1.

Vital Statistics of the United States 1970 Vol. 11.

Figure 2. Age-Adjusted Death Rate from Cardiovascular Disease in 12 Countries, $1970 \cdot 1971$

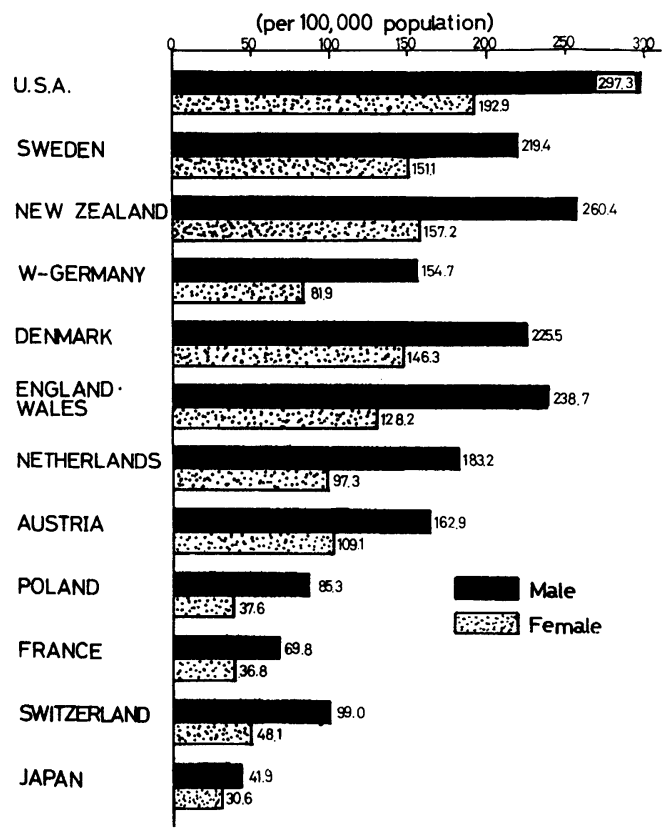

Source :Vital Statistics of Japan.

World Health Statistics Annual, 1972. 
Figure 3. Mortality Trend of Cerebro-and Cardio-Vascular Diseases in Japan

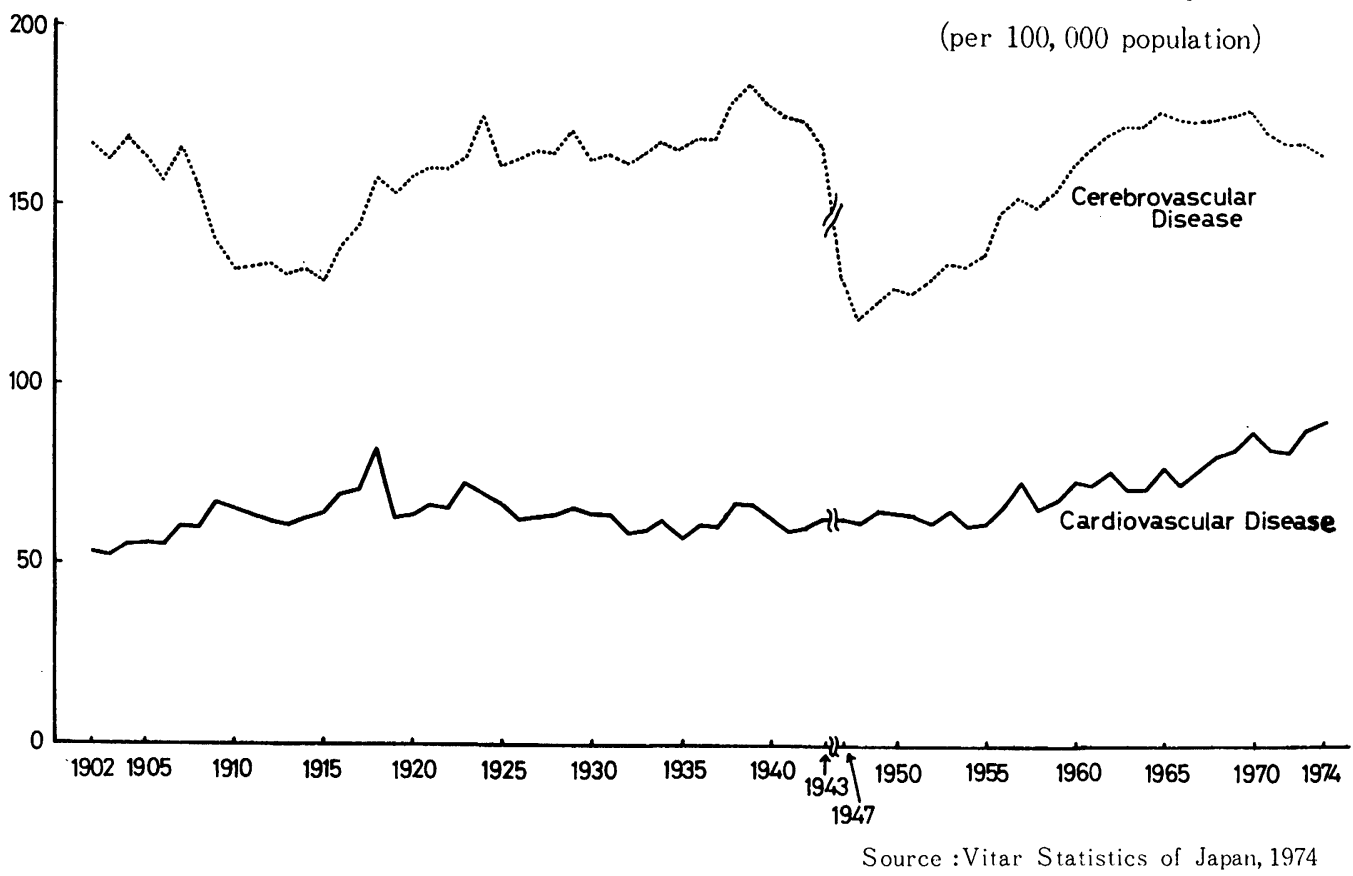

Figure 4. Relative Frequences of Cerebral Hemorrhage and Thrombosis in Japan and Other Countries

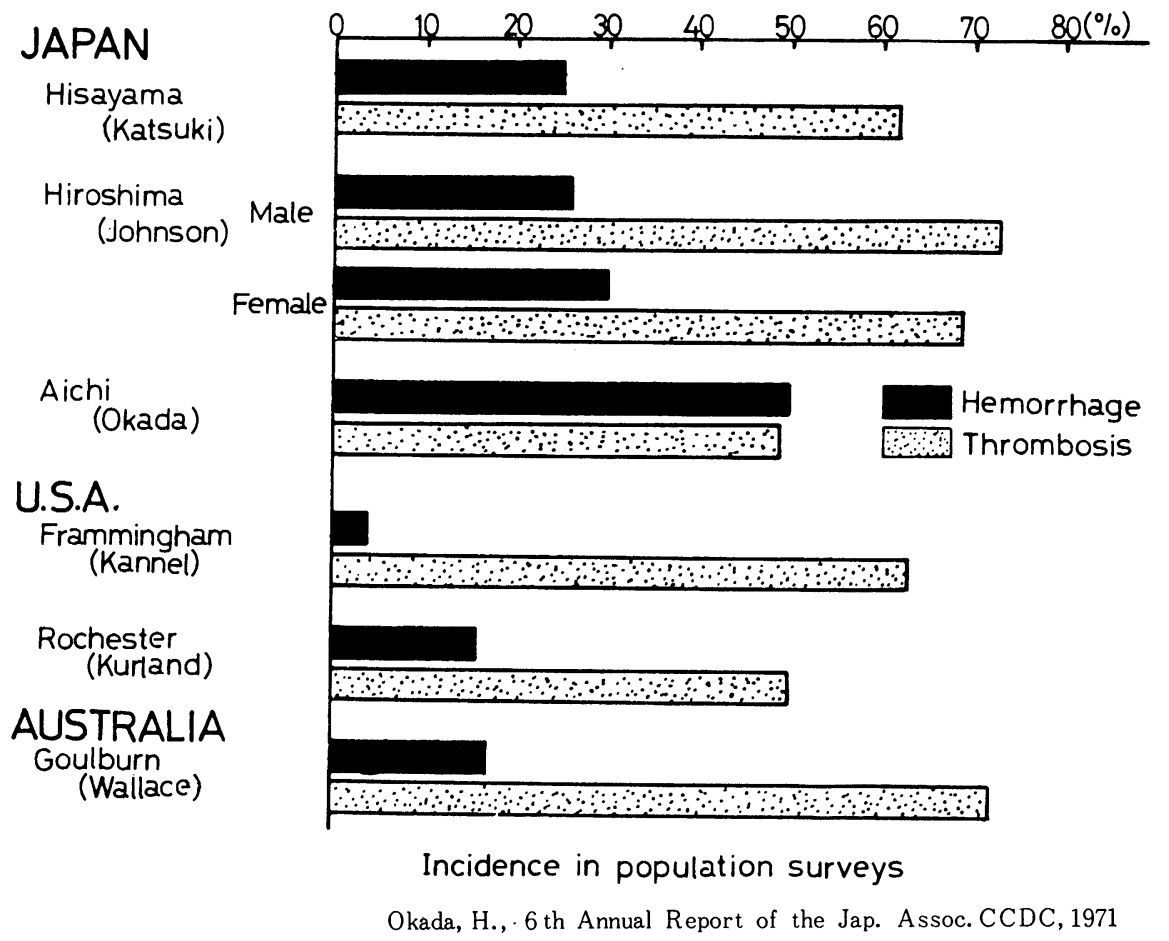



coronary heart disease in Japan compared with European countries.

Figure 5. Cerebrovascular Disease 1970

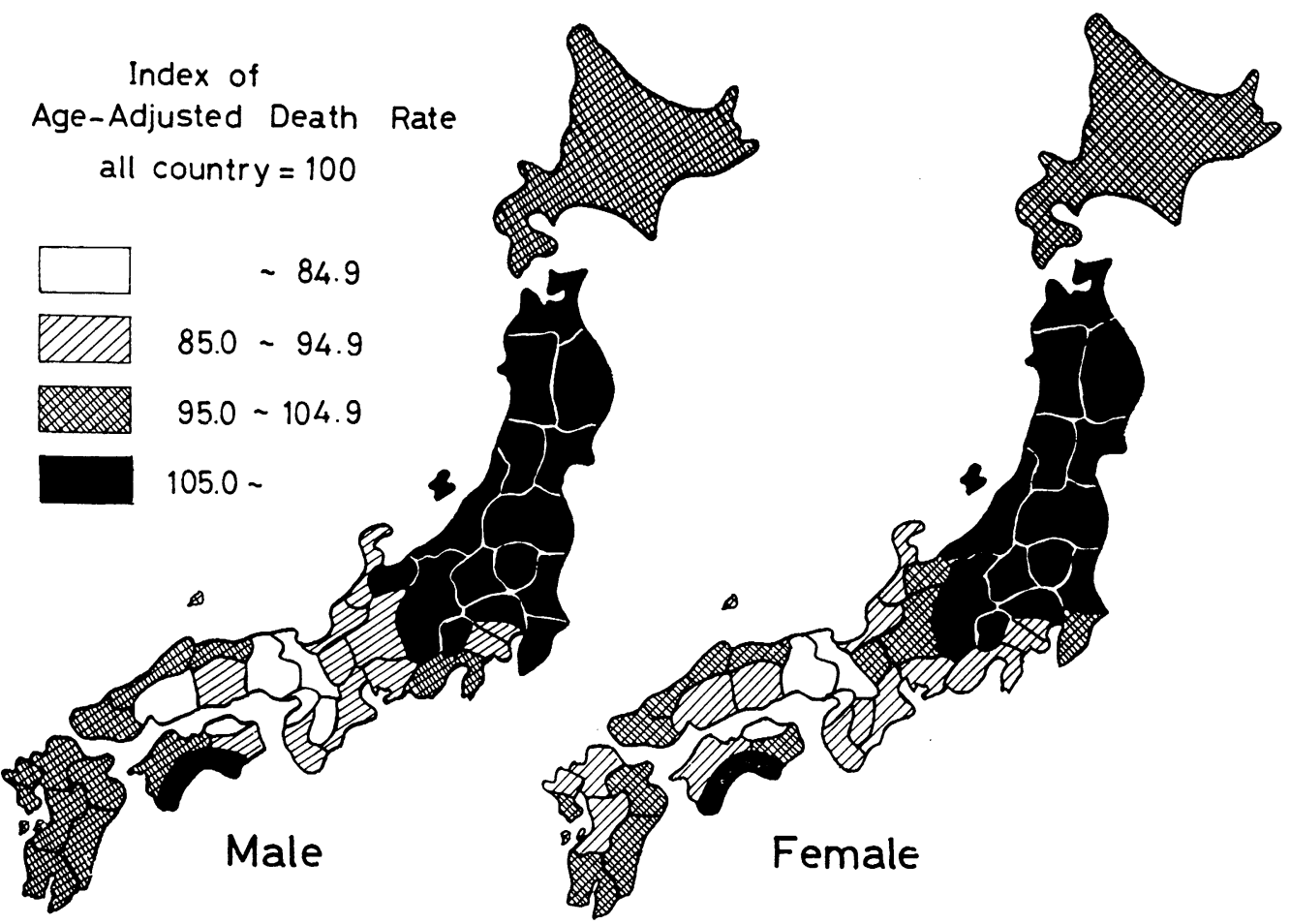

Soures : Ministry of Welfare, Age Adjusted Death Rate in Japan, 1970

Figure 6. Relation of Cerebral Stroke with $\mathrm{NaCl}$ Intake (Males, 40-69 in Age)

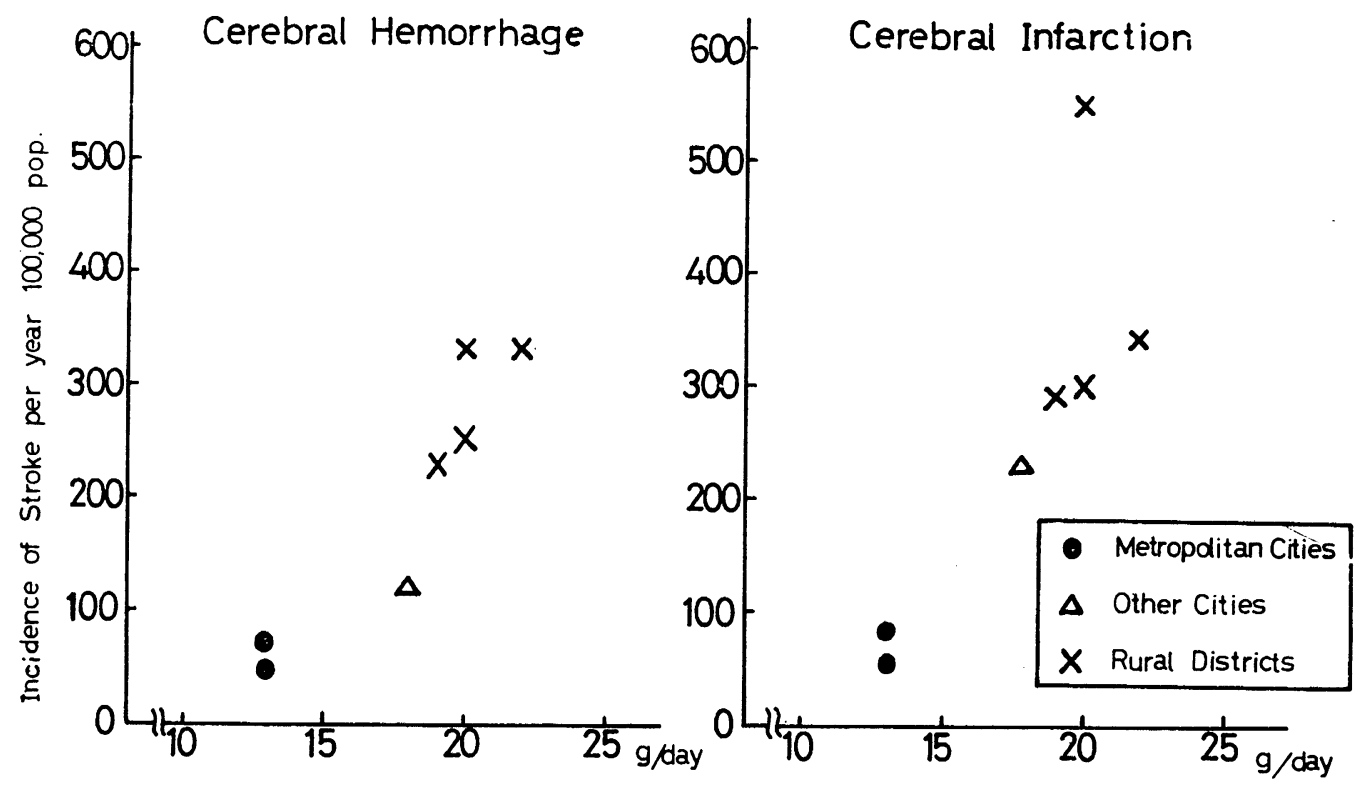

Komachi, Y., et al. Annual Report of the Center for Adult Diseases, 1975. Osaka 
Figure 7. Heart Disease

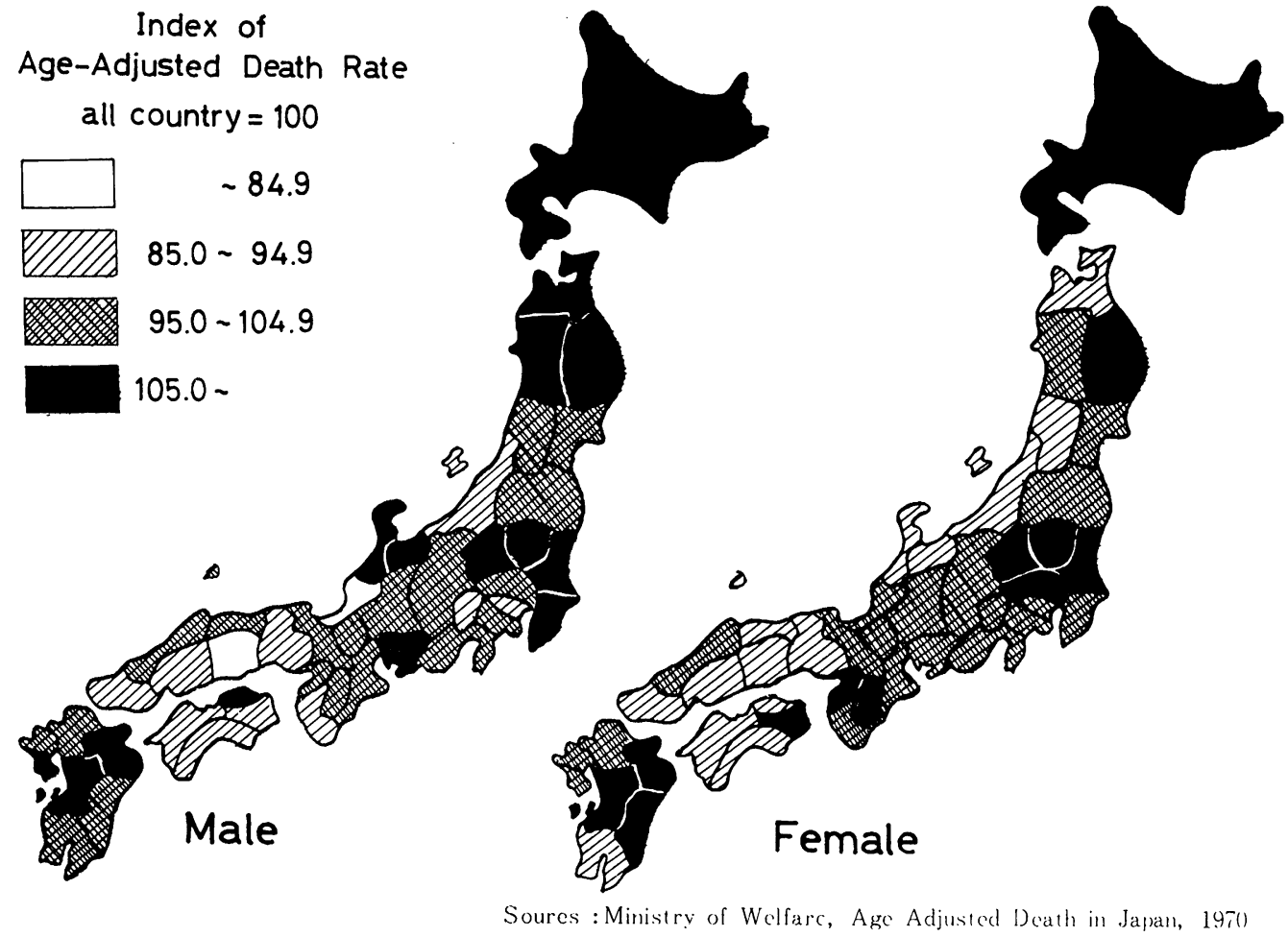

Figure 8. Age-Adjusted Death Rate from Cardiovascular Discase in Japan

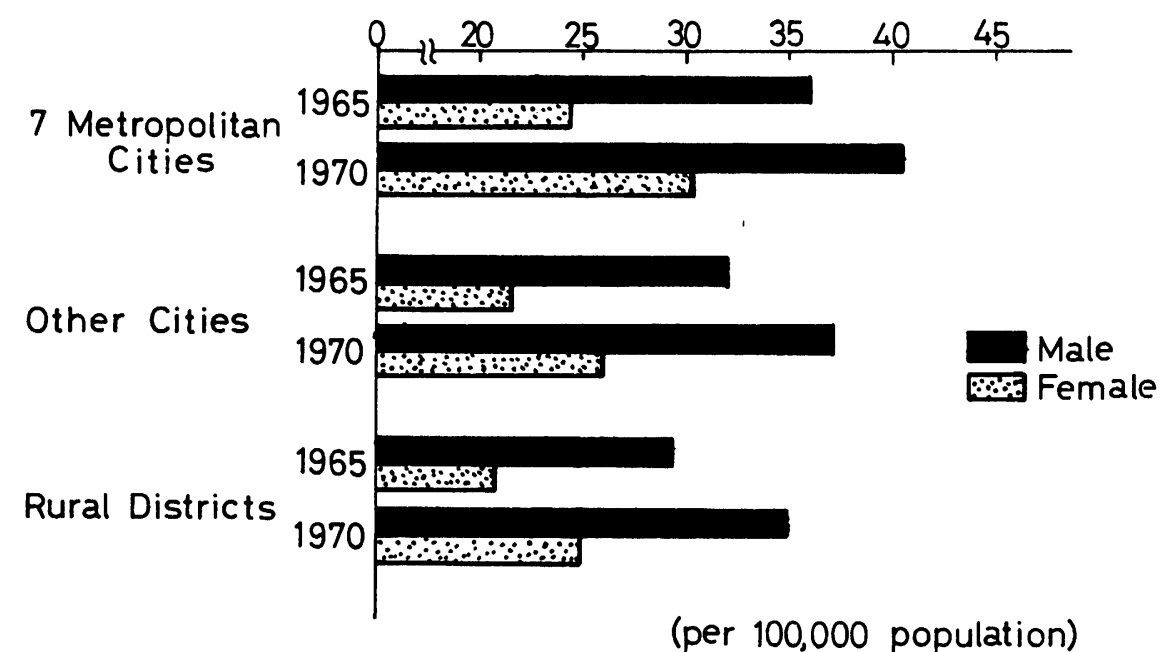


Figure 9. Trend of Nutrient

Total Calories

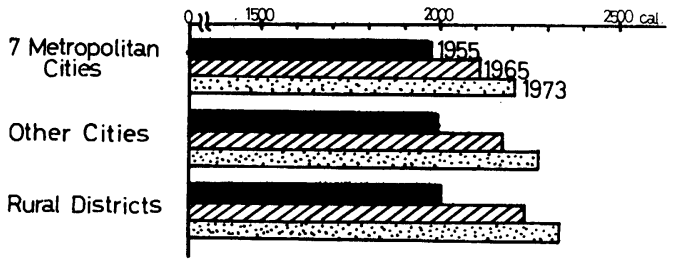

Fat

7 Metropolitan Cities

Other Cities

Rural Districts

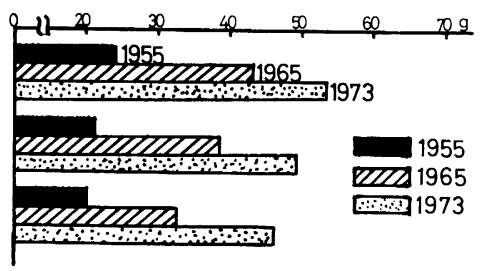

\section{Carbohydrate}

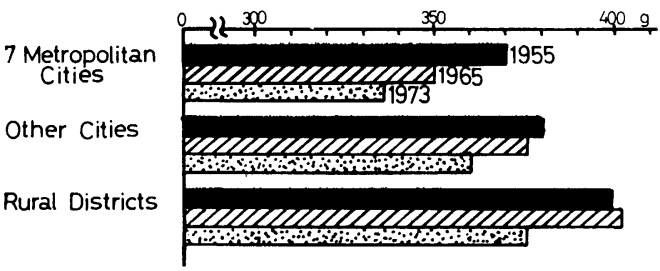

"Present Status of Food Consumption in Japan" Ministry of Welfare, Japan, 1976

Figure 10. Total Serum Cholesterol Level in Japanese Men

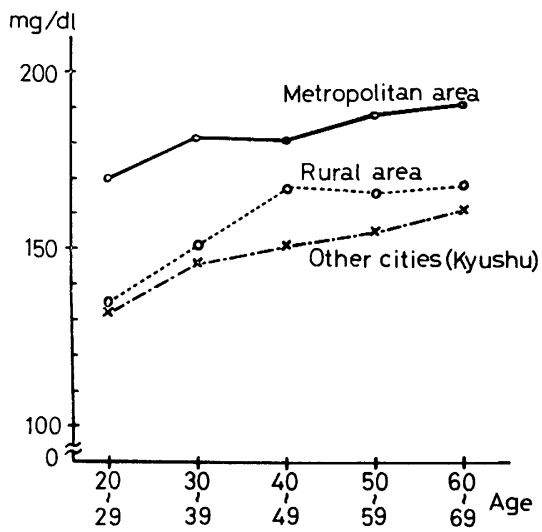

Rescarch Committee on Artherosclerosis in Japan : Jap. Circul. J., Vol. 29, 1965

H.Okada : Jap. Assoc. CCDC., Vo!.6, 1975

\section{References}

1) Okada, H., et al. :A prospective study of cerebrovascular disease in Japanese rural communities, Akabane and Asahi.

Part 1: Evaluation of risk factors in the occurrence of cerebral hemorrhage and thrombosis. Stroke, 7, No. 6, 599-607, 1976.

2) Kurtzke, J. F. : Epidemiology of cerebrovascular disease. Springer-Verlag, Berlin, 1969.

3) Ooneda, G.:Cerebral-hemorrhage of Japanese (Pathological point of view). Igaku-noayumi, Vol. 74, No. 3, 103-107, 1970.

4) Ministry of Health and Welfare :Vital statistics of Japan, 1974. Health and Welfare Statistics Association, 1976.

5) World Health Statistics Annual, Vol. 1, 1971.

6) Vital Statistics of the United States, Vol. 2, 1970.

7) ibid, 1972

8) Okada, H.: On the risk factors of cerebrovascular disease. (Prospective study of three Japanese rural areas). 6th Annual Report of the Japanese Association for Cerebro-cardiovascular Disease Control, 1971.

9) Kat suki, S., et al. : Current concept of the frequency of cerebral-hemorrhage and cerebral infarction in Japan. Cerebral Vascular Diseases, 99-111, 1966.

10) Johnson, K.G., et al. : Cerebral vascular disease in Hiroshima, Japan. J. chron. Dis., 20, 545-559, 1967.

11) Kannel, W. B., et al. : Vascular disease of the brain - Epidemiologic aspects : the Framingham study. A. J. P. H., 55, No. 9 , 1355-1366, 1965. 
12) Kurland, L. T. : Descriptive epidemiology of selected neurologic and myopathic disorders with particular reference to a survey in Rochester, Minnesota. J. chron. Dis., 8, 378-418, 1958.

13) Wallace, D. C., et al. : Study of the natural history of cerebral vascular disease. Med. J. Austr., 1, 90-95, 1967.

14) Ministry of Health and Welfare : Age adjusted death rate in Japan-Main causes, 1970. Health and Welfare S tatistics Association, 1974.
15) Shigiya, K., Komachi, Y., and Watanabe, T. The relation between nutrition and circulatory disєases in Japan. Hokendozin-sha, 1976.

16) Ministry of Health and Welfare : Present status of food consumption in Japan, 1975.

17) Research Committee on Artherosclerosis in Japan : Jap. Circul. J., 29, 1965.

Presented at the 7 th European Congress of
Cardiology which was held in Amsterdam,
June 20-25, 1976. 